\title{
THE INTERACTION OF HUMAN OSTEOBLAST-LIKE Saos-2 CELLS WITH STAINLESS STEEL AND Si(100) COATED BY SILICALITE 1 FILMS
}

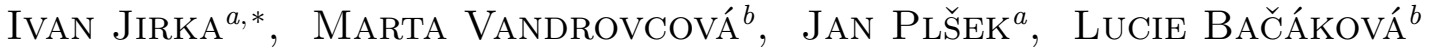 \\ ${ }^{a}$ Heyrovský Institute of Physical Chemistry, ASCR, v.v.i. Dolejškova 3, 18223 Prague 8, Czech Republic \\ ${ }^{b}$ Institute of Physiology, ASCR, v.v.i., Vídeňská 1083, 14220 Prague 4, Czech Republic \\ * corresponding author: ivan.jirka@jh-inst.cas.cz
}

\begin{abstract}
Interaction of osteoblast-like Saos-2 cells with polished stainless steel coupons and $\mathrm{Si}(100)$ wafers covered by a film of densely intergrown a, b oriented silicalite- 1 crystals is investigated. Due to their unique chemical, mechanical and biological properties these films are promising new anti-corrosive coating for bone replacement. Biocompatibility of silicalite 1 film is affected by its morphology and wettability. The surface properties of silicalite 1 film is controlled by synthesis conditions and postsynthetic modifications. The morphology of the film (number of a oriented crystals) is tuned by time of synthesis. Wettability of the film is tuned by heat treatment in a stream of air at $300{ }^{\circ} \mathrm{C}$. The silicalite 1 film is proved to be biocompatible. The number of adhered Saos-2 cells increases with the number of a oriented crystals observed for the film grown on stainless steel and with increased wettability of heat treated samples. Obtained results demonstrate possible ways of preparation of anti-corrosive silicalite-1 coatings with optimized cytocompatibility.
\end{abstract}

KEYWORDs: Biocompatibility, silicalite-1 film, outer zeolite surface, wettability.

\section{INTRODUCTION}

Metals and metal alloys are nowadays the most frequently used implant materials [1. Their substantial drawback is the evolution of metal species into the body. Great attention has thus been paid to developing suitable surface modifications, which do not lower the biocompatibility of the material and simultaneously suppress evolution of the metal species. Silicalite-1 coating $(S C)$ has been found to be prospective in this area [2].

Various physical-chemical parameters, including wettability and morphology, influence the biocompatibility of prosthetic materials [3]. Possibility the optimization of these parameters by the mode of synthesis and post-synthetic treatments of $S C$ to get its maximal biocompatibility is demonstrated in this paper. Our preliminary data of tuning the wettability and morphology of $S C$ by selection of support and the mode of synthesis are presented.

\section{EXPERIMENTAL}

The $S C s$ were synthesized in situ on the polished surface of stainless steel foil and $\mathrm{Si}(100)$ wafer from reaction mixture of tetrapropyl-ammonium hydroxide (TPA, 1M solution in H2O, Sigma Aldrich), tetraethylorthosilicate (TEOS, $\geq 99.0 \%$, Aldrich) and deionized water as described in [4]. The reaction mixture was aged for 2 hours. The synthesis proceeded 3 hours ( $S C$ on $\mathrm{Si}(100)$, referred as to $S C$-Si(100)) and 6 hours ( $S C$ on stainless steel, $S C$-ss) at $165^{\circ} \mathrm{C}$. The supports and synthesis conditions used enabled the preparation of the samples with substantially different surface morphologies. A portion of the synthesized samples were heated at $300{ }^{\circ} \mathrm{C}$ in a stream of dry air. The heattreated samples were abbreviated as $S C$-Si(100)-300 and $S C$-ss-300.

Static water drop contact angle $\theta$ measurements using the SEE system (Brno, Masaryk University, Czech Republic) were utilized to characterize the wettability of $S C$. The morphology of the samples was characterized by near-field scanning electron microscopy (FESEM, S 4800-1, Hitachi).

The human osteoblast-like Saos-2 cells were seeded on the sample surfaces as described in our earlier study [5]. Briefly, the samples were sterilized in a hot air sterilizer $\left(2\right.$ hours, $\left.120^{\circ} \mathrm{C}\right)$, inserted into 24 well cell culture plates, and seeded with the cells at a density of 15,000 cells $/ \mathrm{cm}^{2}$ and in $1 \mathrm{~mL}$ of McCoy's $5 \mathrm{~A}$ medium with $15 \%$ fetal bovine serum and 40 $\mu \mathrm{g} / \mathrm{mL}$ of gentamicin.

\section{RESUlts AND DisCUSSION}

SEM images of the samples $S C$-Si(100) and $S C$-ss are depicted in Figure 1. While the $S C$-Si(100) was mostly composed from a compact layer of intergrown b oriented crystals covered by a small number of a oriented crystals, a high concentration of a oriented crystals on compact layer of intergrown b oriented crystals was observed on the $S C$-ss. No morphological changes were induced upon heating.

Typical results of water drop contact angle measurements are summarized in Figure 2 The $\theta$ values of $S C$-ss and $S C$-Si(100) were comparable; i.e. $84^{\circ} \pm$ $3^{\circ}$ and $66^{\circ} \pm 2^{\circ}$, respectively. Heat treatment induced 


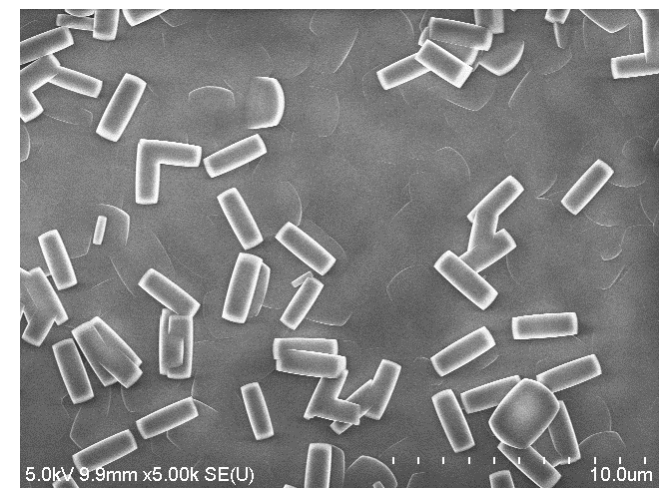

(A).

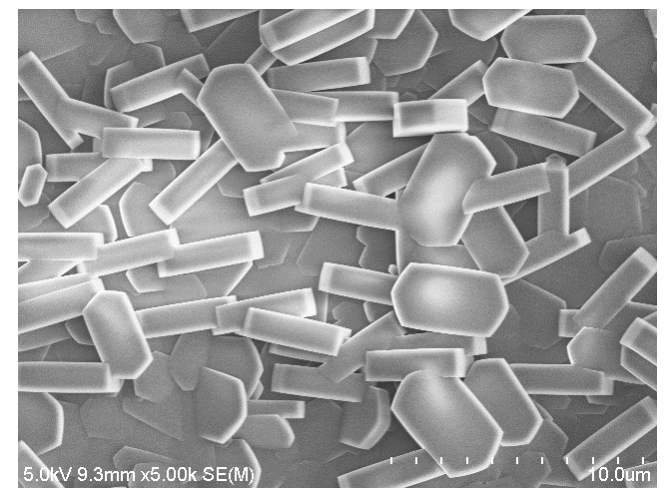

(C).

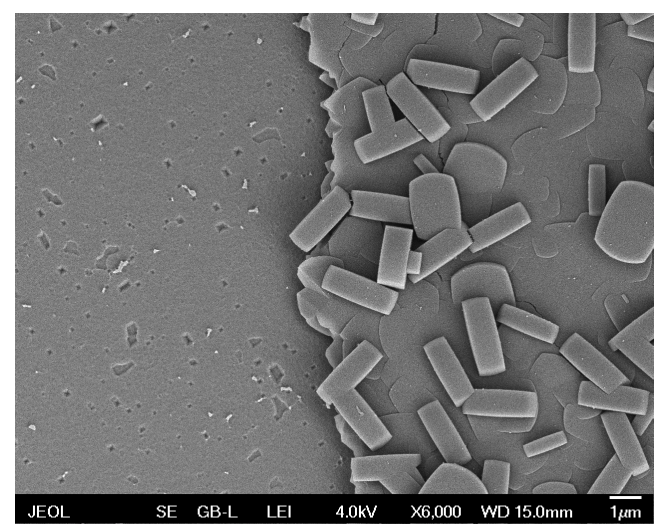

(B).

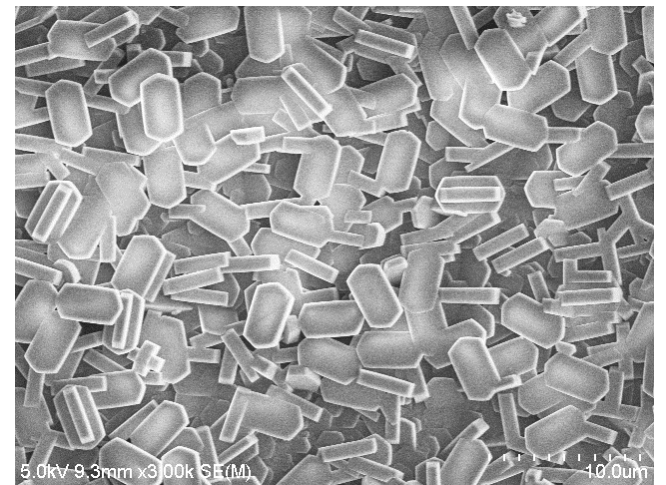

(D).

Figure 1. SEM image of $S C$-Si(100) (top) and $S C$-ss (bottom). Left: a centre of the films: left, right: a periphery of the films.

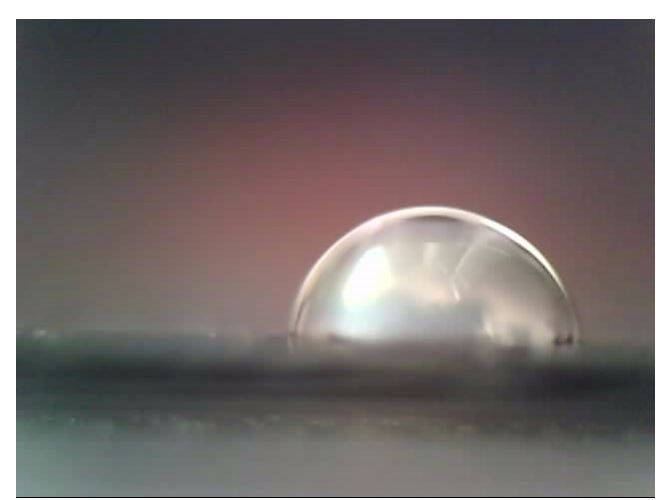

(A).

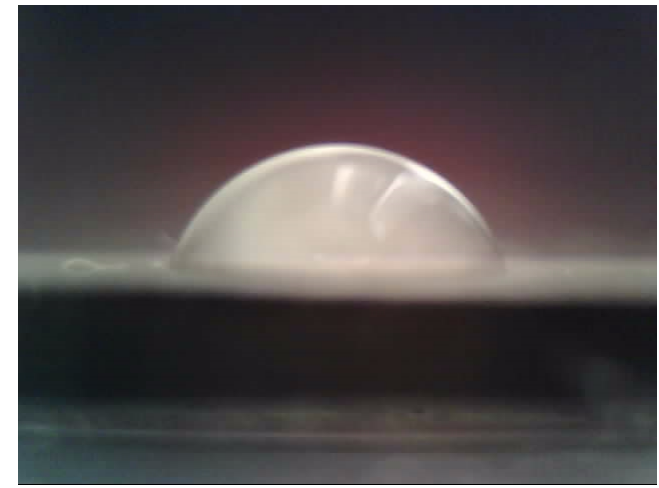

(C).

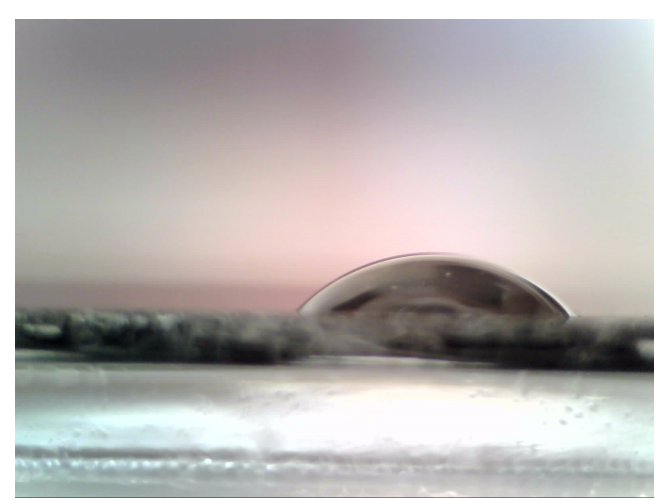

(B).

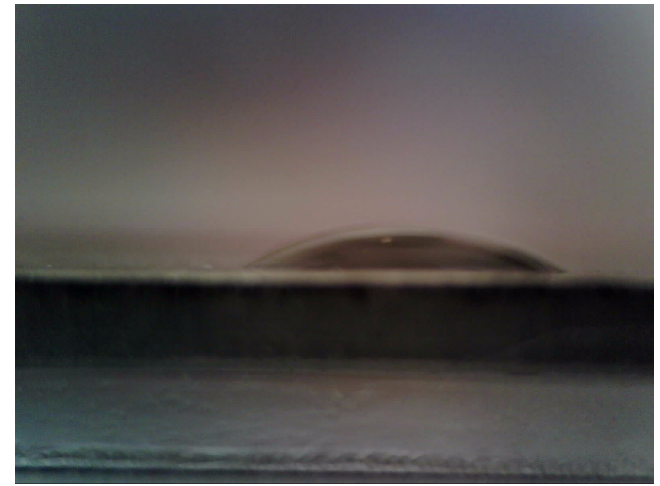

(D).

Figure 2. Water drop contact angle of $S C$-Si(100) (top) and $S C$-ss (bottom) before (left) and after treatment at $300^{\circ} \mathrm{C}$. 


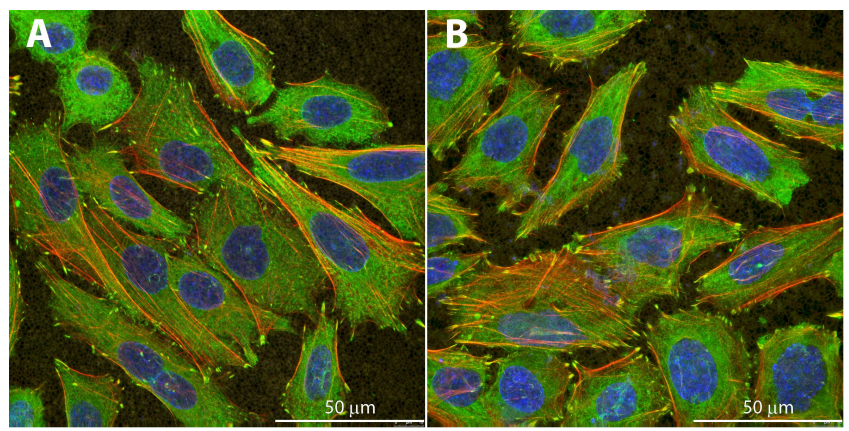

FigURE 3. Immunofluorescence staining of vinculin, a protein of focal adhesion plaques (green) in human osteoblastlike Saos-2 cells on day 3 after seeding on $S C$-Si(100) (A) or $S C$-SS (B). The actin cytoskeleton is stained in red with phalloidin-TRITC, and the cell nuclei are stained in blue with DAPI. Leica TCS SPE DH 2500 confocal microscope.

substantial lowering of the $\theta$ value, which was similar for $S C$-ss and $S C$-Si(100), i.e., $32^{\circ} \pm 3^{\circ}$ and $43^{\circ} \pm 3^{\circ}$, respectively.

The morphology of the Saos-2 cells on the sample $S C$-ss and $S C$-Si(100) is shown in Figure 3.

The cells on both surfaces are of similar morphology, i.e. they are well-spread and polygonal, with welldeveloped vinculin-containing focal adhesion plaques and F-actin cytoskeletons. The number of Saos-2 cells $(N(S a o s-2))$ on the surfaces of $S C$-ss and $S C$-Si(100) on day 1 and 3 after seeding is depicted in Figure 4 The N(Saos-2) on the zeolite samples are compared with the cell numbers on polystyrene dish $(P S)$. On day 1 after seeding, the number on both $S C$ samples is equal ( $S C$-Si(100) or higher $(S C-s s)$ than on $P S$; i.e. the zeolite samples are bioactive. Comparable values of $N($ Saos-2) are observed on the $S C$-ss and $S C$-Si(100) before and after heat treatment of the samples on day 1, after seeding (Figure 4). However, a substantial increase of $N(S a o s-2)$ on $S C$-Si(100)300 and particularly $S C-s s-300$ in comparison with non-heated samples and $P S$ was evident in day-3 after seeding.

\section{Conclusion}

Silicalite-1 films are biocompatible materials. Their interaction with Saos-2 cells is influenced by surface wettability and topography. The number of adhered Saos-2 cells depends on synthesis conditions of zeolite coating and on its post-synthetic modification. The cell number increases with increasing numbers of a oriented zeolite crystals on the surface of $S C$ and increasing surface wettability. Obtained results are prospective in the optimization of $S C$ outer surface to reach its maximal cytocompatibility.

\section{ACKNOWLEDGEMENTS}

Supported by Grant Agency of the Czech Republic (grant No.16-02681S).

\section{REFERENCES}

[1] X. Liu, P. K. Chu, C. Ding. Surface modification of titanium, titanium alloys, and related materials for biomedical applications. Materials Science and

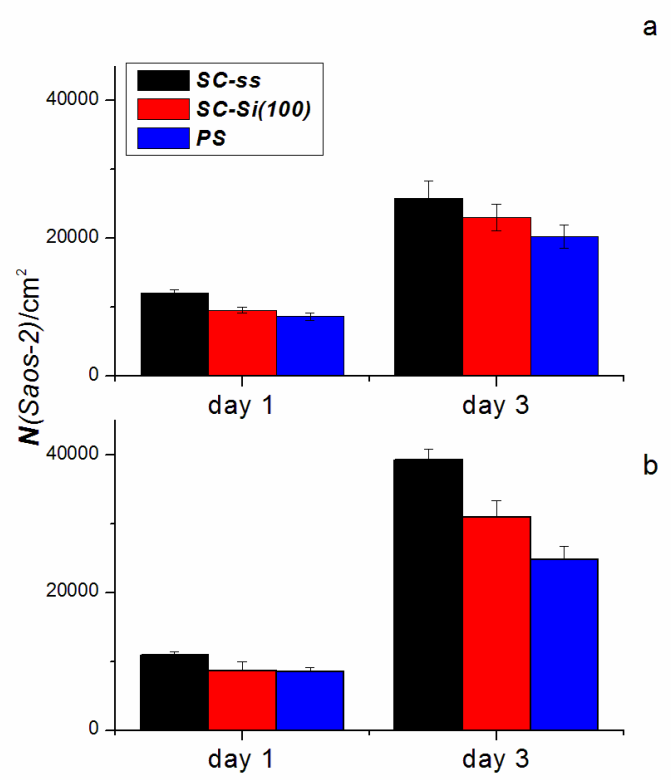

Figure 4. Comparison of the $N($ Saos-2) on days 1 and 3 after seeding on the surfaces of the samples $S C$-SS, SC-Si(100) and PS before (a) and after heat treatment (b) of the samples at $300^{\circ} \mathrm{C}$.

Engineering: R: Reports 47(3-4):49 - 121, 2004. DOI:http://dx.doi.org/10.1016/j.mser.2004.11.001.

[2] R. S. Bedi, D. E. Beving, L. P. Zanello, Y. Yan. Biocompatibility of corrosion-resistant zeolite coatings for titanium alloy biomedical implants. Acta Biomaterialia 5(8):3265 - 3271, 2009. DOI:http://dx.doi.org/10.1016/j.actbio.2009.04.019

[3] L. Bacakova, E. Filova, M. Parizek, et al. Modulation of cell adhesion, proliferation and differentiation on materials designed for body implants. Biotechnology Advances 29(6):739 - 767, 2011. DOI:http://dx.doi.org/10.1016/j.biotechadv.2011.06.004

[4] Z. Wang, Y. Yan. Oriented zeolite $\{$ MFI $\}$ monolayer films on metal substrates by in situ crystallization. Microporous and Mesoporous Materials 48(1-3):229 238, 2001. DOI:http://dx.doi.org/10.1016/S1387-1811(01)00357-2 
[5] M. Vandrovcova, I. Jirka, K. Novotna, et al.

Interaction of human osteoblast-like saos-2 and MG-63

cells with thermally oxidized surfaces of a

titanium-niobium alloy. PLOS ONE 9(6):1-15, 2014.

DOI:10.1371/journal.pone.0100475. 